\title{
Disturbance of peripheral microvascular fluid permeability by the onset of atrioventricular asynchrony in patients with programmable pacemakers
}

\author{
I R Mahy, D M Lewis, A C Shore, M D Penney, L D R Smith, J E Tooke
}

\begin{abstract}
Background-In vitro and in vivo evidence suggests that atrial natriuretic peptide can enhance fluid flux from intravascular to extravascular compartments. The relevance of this to human pathophysiology remains unclear.

Objectives-To determine whether a central haemodynamic change associated with increased plasma concentrations of atrial natriuretic peptide produces detectable change in the capillary filtration coefficient in a peripheral microvascular bed.
\end{abstract}

Patients-12 patients with programmable dual chamber permanent pacemakers.

Methods-Calf capillary filtration coefficient (using a modified plethysmographic technique) and plasma atrial natriuretic peptide concentrations were measured during atrioventricular synchronous and ventricular pacing.

Results-Atrioventricular asynchrony was associated with higher mean (SD) concentrations of atrial natriuretic peptide $(231.9(123.1) v 53.5(38.8) \mathrm{pg} / \mathrm{ml})$ and an increased mean (SD) calf capillary filtration coefficient $(4 \cdot 2)(1 \cdot 1) \quad v \quad 3.6(1 \cdot 1)$ $\mathrm{ml} / \mathrm{min} . \mathrm{mm} \mathbf{H g} .100 \mathrm{ml} \times 10^{-3}$ ), but there was no correlation between the magnitude of the change in these variables in individual patients.

Conclusions-The peripheral capillary filtration coefficient may change in response to altered central haemodynamics. Atrial natriuretic peptide remains one potential candidate mechanism, but other factors are also likely to be involved.

(Heart 1996;75:509-512)

Keywords: capillary filtration; pacing; atrial natriuretic peptide

Despite the considerable capacity of the peripheral microcirculation for autoregulation in health there are reasons to believe that such autoregulation may change in the face of cardiac disease, as a consequence of altered central haemodynamics and the associated neurohumoral changes. One potential mediator of change at a microvascular level is atrial natriuretic peptide, which among other effects has been shown to be capable of altering cardiac filling pressures in man at doses that do not modify renal excretory variables or produce vasodilatation. ${ }^{1}$

It has been suggested that this effect occurs due to enhancement of fluid flux from intravascular to extravascular compartments. This might be caused by modulation of capillary pressure or alteration in capillary hydraulic conductance, with some support for both hypotheses. Atrial natriuretic peptide increases microvessel hydraulic conductance in vitro, ${ }^{2}$ while infusion of atrial natriuretic peptide at physiological doses increases forearm capillary filtration coefficient in humans. ${ }^{3}$ Animal studies have suggested that atrial natriuretic peptide may increase post-capillary resistance, ${ }^{4}$ and that atrial natriuretic peptide may inhibit $\alpha_{1}$ mediated basal tone of large arterioles without altering post-junctional $\alpha_{2}$ adrenoceptors. 5

Though the balance of evidence suggests that atrial natriuretic peptide can alter the capillary filtration coefficient, ${ }^{2367}$ this view is not universally held ${ }^{89}$ Furthermore, the relevance of in vitro and pharmacological studies to human pathophysiology remains unproven. Previous human studies have been predominantly conducted in healthy volunteers, have involved intra-arterial or intravenous infusion, and have produced varying plasma concentrations of atrial natriuretic peptide. The question remains as to whether changes in plasma atrial natriuretic peptide concentrations occurring as a consequence of physiological stimuli are associated with changes in capillary filtration.

Atrioventricular asynchrony has been previously shown to be associated with an increased plasma level of atrial natriuretic peptide, ${ }^{10}$ and can be induced in patients with multiprogrammable dual chamber permanent pacemakers without pharmacological interference. The present study was undertaken to determine whether this central change can produce detectable changes in capillary filtration coefficient in the absence of major haemodynamic consequences. A modified plethysmographic protocol was used for measurement of the capillary filtration coefficient which overcomes many of the theoretical limitations associated with the more conventional technique used in previous studies (vide infra).

Patients and methods PATIENTS

The study comprised 12 patients (seven 


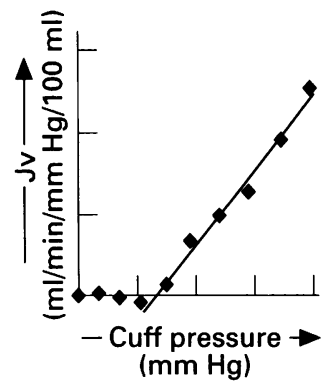

Figure 1 Example of changes in fluid flux (fv) with stepwise increments in cuff pressure in a single individual. The slope of the line provides an estimate of the capillary filtration coefficient and the $x$ axis intercept represents the isovolumetric venous pressure at which neither fluid filtration nor reabsorption occurs.

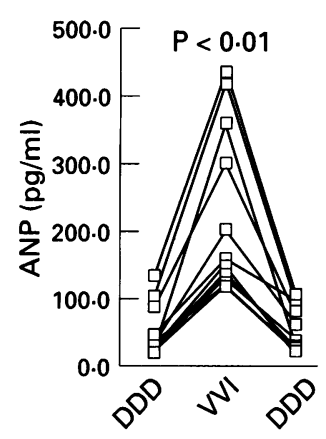

Figure 2 Change in plasma atrial natriuretic peptide ( $A N P$ ) between atrioventricular synchronous (DDD) and asynchronous (VVI) pacing.

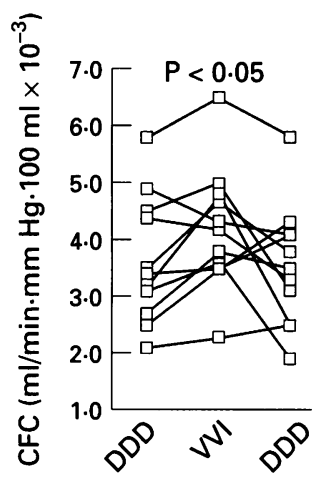

Figure 3 Change in capillary filtration coefficient (CFC) between atrioventricular synchronous (DDD) and asynchronous (VVI) pacing. women, age 26-75 years) with multiprogrammable dual chamber pacemakers who were otherwise in good health. The initial indication for pacing was intermittent or established atrioventricular block in seven patients, sinus node disease in three, and malignant vasovagal syncope as demonstrated by tilt table testing in two. All had preservation of atrioventricular synchrony through normal sinus rhythm or pacing in VDD or DDD modes.

Six patients were taking no medication. One each was taking the combined oral contraceptive pill, hormone replacement therapy, warfarin, propafenone, amlodipine, and nifedipine. Medication remained unchanged throughout the study.

No patient had signs or symptoms of heart failure, peripheral vascular disease, diabetes mellitus, or venous insufficiency of the leg.

\section{MEASUREMENT OF CAPILLARY FILTRATION} COEFFICIENT

The capillary filtration coefficient was measured by mercury in silastic strain gauge plethysmography at the calf using the technique described by Gamble et al, ${ }^{11}$ which examines limb volume changes in response to $a$ series of small pressure increments in an occlusive cuff. This method has several important theoretical advantages over the more conventional single step technique used in many previously described studies, which relies on a series of questionable assumptions. ${ }^{12}$

Patients were studied supine. A pressure cuff with multiple air inlets to permit rapid inflation was applied to the thigh above the knee. Calf swelling rate in response to successive increments in cuff pressure was recorded by a mercury in silastic strain gauge linked through a computer based data logging and analysis system. Serial pressure increments of 8-12 $\mathrm{mm} \mathrm{Hg}$ were applied every $5 \mathrm{~min}$ using an air pump equipped with a multiple resistance air bleed. Once ambient venous pressure is exceeded each increase in cuff pressure produces a characteristic response comprising an initial rapid exponential phase reflecting filling of capacitance vessels and a concurrent linear phase due to fluid efflux from the microcirculation. Each study comprised eight to 10 steps (depending on diastolic blood pressure), with a total study duration of $45-60 \mathrm{~min}$.

Use of sequential small pressure steps considerably reduces the duration of the vascular filling component compared with the use of a single large step (possibly because the venoarteriolar response is not invoked ${ }^{11}$ ) reducing the risk of including this component in analysis of

Summary of changes during ventricular pacing and with restoration of atrioventricular synchronous pacing

\begin{tabular}{lccc}
\hline & $D D D / V D D$ & $V V I(80 \mathrm{bpm})$ & $D D D / V D D$ \\
\hline Heart rate (beats/min) & $68 \cdot 8(7 \cdot 3)$ & $80 \cdot 0(0 \cdot 8) \dagger$ & $67 \cdot 8(7 \cdot 4)$ \\
ANP (pg/ml) & $53 \cdot 5(38 \cdot 8)$ & $231 \cdot 9(123 \cdot 1) \dagger$ & $57.3(33 \cdot 8)$ \\
CFC (ml/min.mm Hg.100 ml $\left.\times 10^{-3}\right)$ & $3 \cdot 6(1 \cdot 1)$ & $4 \cdot 2(1 \cdot 1)^{\star}$ & $3.5(1 \cdot 1)$ \\
Pvi (mm Hg) & $27 \cdot 6(8 \cdot 6)$ & $28.9(7 \cdot 4)$ & $24 \cdot 9(12 \cdot 5)$ \\
MABP (mm Hg) & $97 \cdot 0(8 \cdot 2)$ & $101 \cdot 2(11 \cdot 0)$ & $96 \cdot 2(11 \cdot 7)$ \\
Pv (mm Hg) & $10 \cdot 7(6 \cdot 4)$ & $10 \cdot 7(5 \cdot 0)$ & $8.4(8 \cdot 0)$ \\
\hline
\end{tabular}

$P<0.05,+P<0.01$.

ANP, atrial natriuretic peptide; CFC, capillary filtration coefficient; Pvi, isovolumetric venous pressure; MABP, mean arterial blood pressure; Pv, peripheral venous pressure. the filtration slope. Analysis of multiple pressure steps for each patient permitted a plot of fluid flux against cuff pressure allowing an accurate estimate of capillary filtration coefficient from the slope of the line and obviating the need to make the erroneous assumption that isovolumetric venous pressure is zero. (Isovolumetric venous pressure is the pressure at which there is neither net filtration nor absorption at the microvascular wall (fig 1)). This assumption constitutes an important source of error when using single pressure step techniques. ${ }^{12}$ Furthermore, interventions that alter peripheral microvascular flow (e.g. orthostasis) may alter isovolumetric venous pressure by altering fractional fluid extraction along the capillary and thus local oncotic pressure. ${ }^{13}$ Ambient peripheral venous pressure was estimated by extrapolation of the plot of cuff pressure against asymptotic volume. ${ }^{13}$

The mean (SD) intraindividual coefficient of variation in four normal individuals examined four to eight times over nine months was $11 \cdot 6(3 \cdot 4) \%$.

\section{MEASUREMENT OF ATRIAL NATRIURETIC} PEPTIDE

Blood samples for atrial natriuretic peptide assay were drawn into chilled lithium heparin Vacutainers and centrifuged immediately at $4^{\circ} \mathrm{C}$. Plasma was separated and stored at $-30^{\circ} \mathrm{C}$. Samples were analysed in a single batch using the protocol for plasma extraction and radioimmunoassay described by Penney et al. ${ }^{14}$ Intra-assay coefficient of variation for this procedure (extraction and radioimmunoassay) derived from replicate control plasmas is $6.4 \%$ at a mean plasma atrial natriuretic peptide concentration of $72 \cdot 7 \mathrm{pg} / \mathrm{ml}$.

\section{STUDY PROTOCOL}

Baseline studies were performed without alteration of clinically determined pacemaker variables after $20 \mathrm{~min}$ of supine acclimatisation in a temperature controlled laboratory (22 $\left.(0.5)^{\circ} \mathrm{C}\right)$. Capillary filtration coefficient was measured as previously described. At the end of this period (typically 75-80 min of supine rest in total) blood was drawn for measurement of atrial natriuretic peptide.

The pacemaker was then reprogrammed to VVI (ventricular) pacing at a rate of 80 beats/min. Some 60-75 min after reprogramming the patient returned to the laboratory and after further acclimatisation the study was repeated. This time interval was chosen because previous work has suggested that plasma levels of atrial natriuretic peptide reach a peak 90-120 min after the onset of atrioventricular asynchrony ${ }^{15}$ and it was therefore anticipated that maximal levels of atrial natriuretic peptide would be present during the second measurement of capillary filtration coefficient.

A third similar study was performed between three and 17 days (mean nine) after restoration of the original pacemaker variables. The duration of the effect of atrial natriuretic peptide on capillary hydraulic conductance has not been clearly defined. This time inter- 

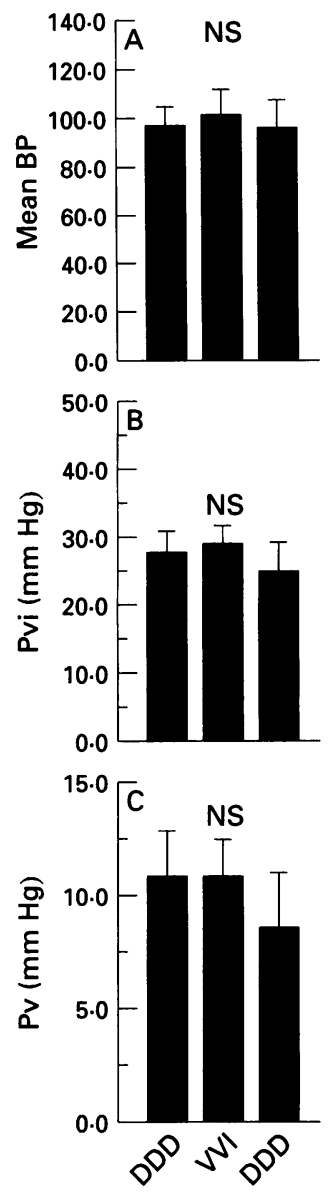

Figure 4 Changes in $(A)$ mean brachial arterial blood pressure $(B P),(B)$ isovolumetric venous pressure (Pvi) and $(C)$ peripheral venous pressure (Pv) between

atrioventricular synchronous (DDD) and asynchronous (VVI) pacing for the group of 11 patients. NS, not significant.

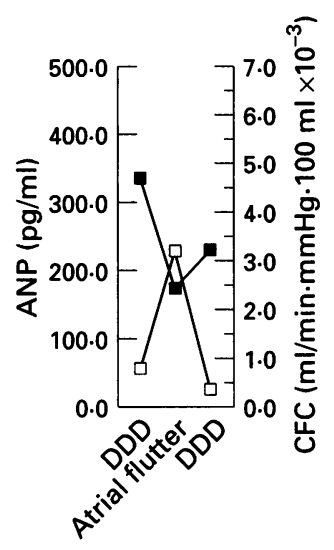

Figure 5 Changes in plasma atrial natriuretic peptide (ANP; $\square$ ) and capillary filtration coefficient (CFC; $\mathbf{D})$ in the patient who developed atrial flutter. val was chosen principally on pragmatic considerations, but was felt sufficient to have allowed the effects of the haemodynamic and associated endocrine changes on the peripheral microcirculation to have resolved.

\section{DATA ANALYSIS}

All plethysmographic data were analysed blind to patient identity and pacing mode. Samples for atrial natriuretic peptide were analysed off site, again blinded to patient identity and pacing mode.

Data are expressed as mean (SD). Group comparisons were undertaken using analysis of variance for repeated measures followed by paired $t$ test where significant. The relation between changes in the capillary filtration coefficient and in atrial natriuretic peptide was examined using Pearson's correlation.

\section{ESTIMATE OF SAMPLE SIZE}

The study of Groban et $a l^{3}$ suggested that increasing plasma levels of atrial natriuretic peptide by four to fivefold produced an increase in capillary filtration coefficient of $37-63 \%$ in healthy controls. On the basis of our own previously reported studies using the plethysmographic protocol described ${ }^{16}$ it was estimated that 10 patients were required to have $90 \%$ power to demonstrate a $25 \%$ change in capillary filtration coefficient (at a $5 \%$ level of significance).

\section{Results}

One patient developed symptomatic atrial flutter with 2:1 atrioventricular block after reprogramming and was therefore excluded from the group analysis. Data from this patient are presented independently.

The remaining 11 patients tolerated ventricular pacing well. Mean atrioventricular interval at baseline was $162.3(20.2) \mathrm{ms}$. During ventricular pacing there was complete atrioventricular dissociation in seven and retrograde ventriculoatrial conduction in four.

With ventricular pacing there was no significant change in mean arterial blood pressure, peripheral venous pressure, or the isovolumetric venous pressure. The concentration of atrial natriuretic peptide rose about fourfold and there was a small but statistically significant increase in the capillary filtration coefficient. These changes were reversed on restoration of atrioventricular synchrony (table and figs 2-4).

No correlation was seen between either the absolute or relative change in plasma atrial natriuretic peptide and the increase in capillary filtration coefficient.

A substantial increase in the concentration of atrial natriuretic peptide was also seen in the patient who developed atrial flutter, but the capillary filtration coefficient behaved differently, decreasing by about $50 \%$ (fig 5 ).

\section{Discussion}

These results support the hypothesis that an acute change in central haemodynamics asso- ciated with increased concentrations of plasma atrial natriuretic peptide may influence peripheral microvascular fluid permeability in the absence of significant changes in arterial blood pressure. However, the change in the capillary filtration coefficient was modest despite substantial changes in atrial natriuretic peptide. Furthermore, the lack of any correlation between the magnitude of the changes in the capillary filtration coefficient and atrial natriuretic peptide and the findings in the patient who developed atrial flutter suggest that in vivo the situation is more complex than implied by previous pharmacological studies and that other factors-perhaps, for example catecholamines-may counterbalance the effects of atrial natriuretic peptide. Nevertheless, the demonstration that microvascular fluid permeability may vary in this way has important implications for the control of other Starling's forces within the tissues, to permit preservation of the balance between tissue fluid economy and nutritive flow in the microcirculation during central haemodynamic change.

Changes in the capillary filtration coefficient may arise as a consequence of a change in either capillary hydraulic conductance or functional capillary surface area. However, the technique used in the present study to measure capillary filtration probably takes account of all anatomically available capillaries due to "back perfusion" at the increased venous pressures applied. ${ }^{17}$ Possible functional changes in capillary surface area may therefore be less relevant to capillary filtration coefficient measured in this way than those in capillary hydraulic conductance.

An alternative explanation for the findings presented is that haemodynamic or neuroendocrine changes influence the relation between venous pressure and capillary pressure during cuff inflation through effects on the ratio of pre- to post-capillary resistance. Although such a possibility cannot be entirely excluded, it is unlikely because the multistep plethysmographic protocol used (which allows the capillary filtration coefficient to be derived from increments in venous pressure within a higher range of venous pressures) would largely negate the impact of any such effect; firstly, because the capillary filtration coefficient is derived from multiple changes in venous pressure and secondly, because at higher starting values of venous pressure errors introduced by changes in pre- to post-capillary resistance are minimised. ${ }^{12}$

In vitro evidence suggests that atrial natriuretic peptide can alter hydraulic conductivity of single microvessels. ${ }^{2}$ Although the present study failed to show a direct relation between the magnitude of changes in atrial natriuretic peptide and those in the capillary filtration coefficient this may reflect inhomogeneity within the patient group studied. For example, our patients covered a wide age range and had a variety of indications for pacing. These differences are possibly associated with variations in the response of the microvascular bed. Alternatively, it remains possible that changes 
in the capillary filtration coefficient seen in the present study are mediated by atrial natriuretic peptide, but arise through a more complex mechanism in vivo than suggested by in vitro work. For example, it has been suggested that changes in baroreceptor function in patients with mild heart failure may modulate capillary filtration coefficient ${ }^{18}$ and atrial natriuretic peptide may in turn influence baroreceptor responses. ${ }^{19}$

The relative increase in atrial natriuretic peptide achieved by transition from atrioventricular synchronous to asynchronous pacing in this study is greater than identified in some previous studies. ${ }^{20} 21$ This may in part reflect the fact that at baseline the mean atrioventricular interval in the present study approached $175 \mathrm{~ms}$, which has been shown to be associated with lower levels of atrial natriuretic peptide than shorter or longer atrioventricular intervals. ${ }^{22}$ Further, the relative change in atrial natriuretic peptide on switching from atrioventricular synchronous to asynchronous pacing has been shown to be greater at lower heart rates such as that used in the current study. ${ }^{10}$

In summary, this study suggests that acute changes in central haemodynamics can influence microvascular fluid permeability in human peripheral tissues, thereby having the potential to alter the rate of fluid transfer across the microvascular wall. This change is coincident with a substantial change in atrial natriuretic peptide, which in conjunction with previous pharmacological studies adds weight to evidence for a possible physiological role for atrial natriuretic peptide in this context. However, further work is required before this link is established and to define its clinical relevance.

This work was supported by The British Heart Foundation.

1 Groban L, Ebert TJ, Kreis DU, Skelton MM, Van Wynsberghe DM, Cowley AW. Haemodynamic, renal, and hormonal responses to incremental ANF infusions in humans. Am F Physiol 1989;256:F780-6.

2 Huxley VH, Tucker VL, Verburg KM, Freeman RH. Increased capillary hydraulic conductivity induced by atrial natriuretic peptide. Circ Res 1987;60:304-7.
3 Groban L, Cowley AW, Ebert TJ. Atrial natriuretic peptide augments forearm capillary filtration in humans. Am $\mathrm{f}$ Physiol 1990;259:H258-63.

4 Chien YW, Frohlich ED, Trippodo NC. Atrial natriuretic peptide increases resistance to venous return in rats. $A m \mathcal{F}$ peptide increases resistance
Physiol 1987;252:H894-9.

5 Faber JE, Gettes DR, Gianturco DP. Microvascular effects of atrial natriuretic factor: interaction with alpha $a_{1}$ - and alpha ${ }_{2}$-adrenoceptors. Circ Res 1988;63:415-28.

6 Ando S, Imaizumi T, Harada S, Hirooka Y, Takeshita A. Atrial natriuretic peptide increases human capillary filtration and venous distensibility. $\mathcal{F}$ Hypertens 1992;10: 451-7.

7 Wijeyaratne CN, Moult PJ. The effect of alpha human atrial natriuretic peptide on plasma volume and vascular permeability in normotensive subjects. $\mathcal{f}$ Clin Endocrinol Metab 1993;76:343-6.

8 Rolleke T, Berke B, Arndt JO. Atrial natriuretic peptide alters neither capillary filtration nor vascular compliance of both skin and skeletal muscle of humans. Basic Res Cardiol 1994;89:192-205.

9 Doorenbos CJ, Blauw GJ, van Brummelen P. Arterial and venous effects of atrial natriuretic peptide in the human vorearm. Am $\mathcal{f}$ Hypertens 1991;4:333-40.

10 Noll B, Krappe J, Goke B, Maisch B. Influence of pacing mode and rate on peripheral levels of atrial natriuretic mode and rate on peripheral level
peptide. $P A C E$ 1989;12:1763-8.

11 Gamble J, Gartside IB, Christ F. A reassessment of mercury in silastic strain gauge plethysmography for microvascular permeability assessment in man. $\mathcal{F}$ Physiol (Paris) 1993;464:407-22.

12 Michel CC, Moyses C. The measurement of fluid filtration in human limbs. In: Tooke JE, Smaje LH, eds. Clinical investigation of the microcirculation. Boston: Martinus Nijhoff, 1987:103-26.

13 Gamble J, Christ F, Gartside IB. Mercury in silastic strain gauge plethysmography for the clinical assessment of the microcirculation. Postgrad Med $\mathcal{F}$ 1992;68:S25-33.

14 Penney MD, Hampton D, Oleesky DA, Livingstine C, Mulkerrin E. Radioimmunoassays of arginine vasopressin and atrial natriuretic peptide: application of a common protocol for plasma extraction using Sep-Pak C18 cartridges. Ann Clin Biochem 1992;29:652-8.

15 Abe Y, Kadowaki K, Sato T, Nakagomi A, Kumagai T. Secretion of atrial natriuretic peptide during artificial pacing: assessments including the influence of

16 Mahy IR, Shore AC, Smith LDR, Tooke JE. Peripheral microvascular function in atrial fibrillation: preservation of capillary pressure and capillary filtration coefficient. of capillary pressure and capillary
Cardiovasc Res $1994 ; 28: 1555-8$.

17 Jaap AJ, Shore AC, Gartside IB, Gamble J, Tooke JE. Increased microvascular fluid permeability in young type 1 (insulin-dependent) diabetic patients. Diabetologia 1993; 36:648-52.

18 Jacobsen TN, Kassis E, Amtorp O. Effects of orthostatic stress on peripheral capillary filtration in mild congestive heart failure after healing of myocardial infarction. $A m \mathcal{F}$ Cardiol 1993;72:418-22.

19 Volpe M, Lembo G, Condorelli G, Luca N, Lamenza F, Indolfi $\mathrm{C}$, et al. Converting enzyme inhibition prevents the effects of atrial natriuretic factor on baroreflex responses in humans. Circulation 1990;82:1214-21.

20 Stangl K, Weil J, Seitz K, Laule M, Gerzer R. Influence of AV synchrony on the plasma levels of atrial natriuretic peptide in patients with total AV block. PACE 1988;11: peptide in

21 Theodorakis GN, Kremastinos DT, Markianos M, Livanis E, Karavolias G, Toutouzas PK. Total sympathetic activity and atrial natriuretic factor levels in VVI and DDD pacing with different atrioventricular delays during daily activity and exercise. Eur Heart $\mathcal{f} 1992 ; 13: 1477-81$.

22 Noll B, Krappe J, Goke B. Modification of atrial natriuretic factor by the AV transmission time in pacemaker factor by the AV transmission time in pacemaker
patients. Deutsche Medizinische Wochenschrift 1988;113: patients. 